Article

\title{
Energy-Efficient Resource and Power Allocation for Underlay Multicast Device-to-Device Transmission
}

\author{
Fan Jiang ${ }^{1, *}$ (D), Honglin Wang ${ }^{1}$, Hao Ren ${ }^{1}$ and Shuai $\mathrm{Xu}^{2}$ \\ 1 Shaanxi Key Laboratory of Information Communication Network and Security, Xi'an University of Posts \\ and Telecommunications, Xi'an 710121, China; wanghonglin928@gmail.com (H.W.); \\ 18591970531@163.com (H.R.) \\ 2 College of Liberal Arts; Xi'an University of Finance and Economics, Xi'an 710121, China; xushuai.8@163.com \\ * Correspondence: jiangfan@xupt.edu.cn
}

Received: 20 October 2017; Accepted: 8 November 2017; Published: 14 November 2017

\begin{abstract}
In this paper, we present an energy-efficient resource allocation and power control scheme for D2D (Device-to-Device) multicasting transmission. The objective is to maximize the overall energy-efficiency of D2D multicast clusters through effective resource allocation and power control schemes, while considering the quality of service (QoS) requirements of both cellular users (CUs) and D2D clusters. We first build the optimization model and a heuristic resource and power allocation algorithm is then proposed to solve the energy-efficiency problem with less computational complexity. Numerical results indicate that the proposed algorithm outperforms existing schemes in terms of throughput per energy consumption.
\end{abstract}

Keywords: Device-to-Device (D2D) communication; multicast transmission; energy-efficiency

\section{Introduction}

With the emergence and popularity of the Internet of Things (IoT) [1,2], billions of devices will be connected and serviced by current wireless networks. In particular, the local area service of popular content sharing is one of the main reasons for this tremendous growth. Such unprecedented growth of data has brought great pressure to current network architectures and technologies. Under this circumstance, the direct connectivity between mobile devices, namely, Device-to-Device (D2D) communication underlay cellular networks, emerges as a potential component for the fifth generation (5G) mobile networks [3].

The concept of underlay D2D multicast transmission refers to the high spectrum-efficient D2D multicast transmission scenario (from the cluster head $(\mathrm{CH})$ to multiple member user equipment (UEs)) which reuses the resource of existing cellular links [4]. By exploiting the inherent broadcast nature of wireless channels, D2D multicast transmission provides an effective solution to offload the heavy data traffic to D2D links, which not only mitigates the burden of the base station (BS) but also increases the spectrum efficiency of the network [5]. Concerning with current researches about D2D multicast transmission, most works mainly focus on utilizing D2D multicast to improve the system spectrum efficiency, or to provide offloading function while the energy efficiency of D2D multicast transmission has often been omitted [6-11]. In this paper, based on the existing contributions, we propose an energy-efficient resource allocation and power control strategy for D2D multicast transmission scenario. Specifically, in order to maximize the overall energy-efficiency of D2D multicast clusters, we first formulate the energy-efficiency optimization problem which is a non-convex problem. Then, we propose a heuristic resource allocation and power control algorithm, which brings computational complexity compared with the conventional exhaustive searching based algorithms. The proposed scheme has better performance with respect to energy-efficiency. 
The rest of the paper is organized as follows. In Section 3, we formulate the network model and illustrate the energy-efficiency maximization problem. Section 4 investigates the resource and power allocation problem with energy-efficient consideration and a heuristic algorithm is then proposed. Simulation results and analysis are given in Section 5. Finally, Section 6 concludes the paper.

\section{Related Work}

Recently, investigations about underlay D2D multicast transmission mainly concentrate on how to mitigate the reuse interference introduced by D2D transmission [6-8]. For example, in order to maximize the total throughput of CUs and D2D clusters in a cellular cell, Meshgi et al. [6] studied a joint channel and power allocation strategy. The work utilizes the maximum weight bipartite matching method to find the optimal resource allocation and power allocation between CUs and D2D pairs. Bhardwaj et al. [7] proposed a scheme to minimize the interference among D2D links and CUs through a resource allocation scheme. The object is to maximize the total throughput of CUs and D2D users through a joint power and channel allocation scheme. Kitagawa et al. [8] proposed an efficient transmitter user selection algorithm, which improves system capacity while minimizing the impact of interference among D2D multicast communications. However, it can be easily seen that most of the above works focus on how to improve the system spectrum efficiency of D2D multicasting while the energy-efficiency aspect of D2D multicasting is not properly addressed.

On the other hand, although some studies already deal with the energy-efficiency aspect of D2D transmission, their research focuses are different from ours. In [9], D2D multicast transmission is suggested to perform the computation offloading task for interactive applications. The proposed algorithm aims at minimizing the energy consumption of each mobile terminal other than the overall energy-efficiency. In [10], a joint power and resource allocations scheme is proposed for D2D underlay multicast communication. This work mainly focuses on how to accommodate more D2D multicast groups while minimizing the total terminal transmission power. In [11], a D2D crowd framework for 5G mobile edge computing is proposed. The authors first introduced the concept of D2D crowd framework, then propose a graph matching-based optimal task assignment policy to address the energy efficient D2D task assignment problem. By taking the energy constraint into account, this work mainly deals with D2D crowd task assignment problem, while the energy-efficiency of the D2D clusters is not considered.

\section{System Model and Problem Formulation}

Without loss of generality, we assume that several D2D clusters have already been formed either by the BS coordinately or by the cluster head in a distributed way, as shown in Figure 1. In fact, D2D cluster formation methods have been widely discussed in existing works, such as [12]. Hence, this paper mainly focuses on cluster-based D2D multicast transmission underlying uplink cellular networks. As illustrated in Figure 1, supposes there are $M$ D2D multicast clusters who share uplink resource blocks (RBs) with $N$ CUs. We use $m, m \in \mathcal{M}=\{1,2, \ldots, M\}$ to index $m$-th D2D cluster, where $\mathcal{M}$ is the set of D2D multicast clusters. To clarify computation, the resource allocation for CUs is assumed to be pre-determined (e.g., the $n$-th RB is allocated to the $n$-th CU). Let $n, n \in \mathcal{N}=\{1,2, \ldots, N\}$ indicates the $n$-th CU and also the RB it occupies, where $\mathcal{N}$ represents the set of CUs. This paper mainly focuses on the matching of D2D multicast clusters and CU as well as the power control policies correspondingly. Within each D2D multicast cluster, the cluster head serves as the D2D transmitter and the cluster members are D2D receivers. Suppose $\mathcal{K}_{m}$ is used to represent the set of receivers in the $m$-th D2D multicast cluster, where $\left|\mathcal{K}_{m}\right|$ denotes the total number of receivers in the $m$-th D2D cluster. When $\left|\mathcal{K}_{m}\right|=1$, the transmission scenario becomes unicast transmission. 


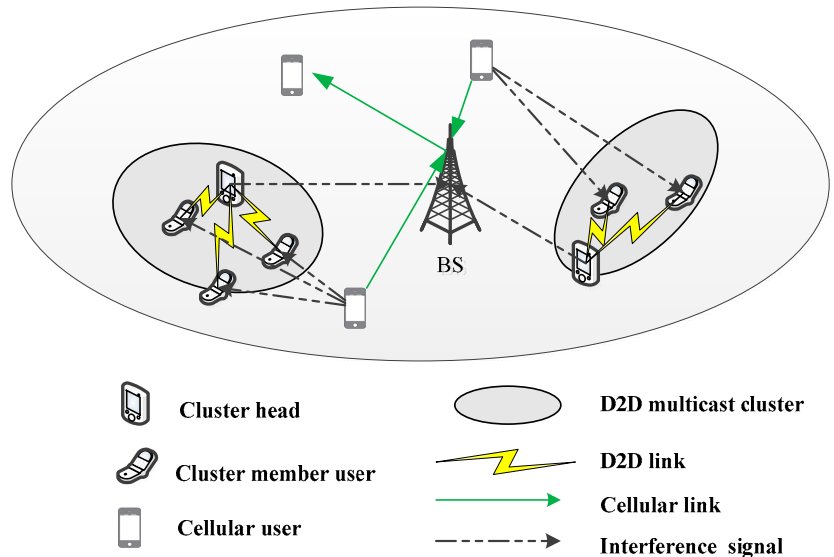

Figure 1. The considered device-to-device (D2D) multicast transmission network model. BS: base station.

We define a set of binary variables $x_{m, n}$. If the $m$-th $\mathrm{D} 2 \mathrm{D}$ cluster reuses the RB of $n$-th CU, then $x_{m, n}=1$, otherwise, $x_{m, n}=0$. Assume each D2D multicast cluster is allowed to reuse at most one RB. Then we have

$$
\begin{cases}\sum_{n=1}^{N} x_{m, n} \leq 1, \quad \forall m \in \mathcal{M} \\ \sum_{m=1}^{M} x_{m, n} \leq 1, \quad \forall n \in \mathcal{N}\end{cases}
$$

Equation (1) indicates two aspects. One is that each D2D multicast cluster is allowed to reuse at most one $\mathrm{RB}$ of a $\mathrm{CU}$; the other is the $\mathrm{RB}$ of a $\mathrm{CU}$ can only be reused by at most one D2D cluster. Consequently, assume the $n$-th RB is reused by $m$-th D2D cluster, the channel quality of the $k$-th member UE who act as a receiver in $m$-th $\mathrm{D} 2 \mathrm{D}$ cluster is given by

$$
\gamma_{k}^{D 2 D_{m}}=\frac{G_{m, k}^{D 2 D}}{\sum_{n=1}^{N} x_{m, n} P_{n}^{C U} G_{n, k}^{C 2 D}+\sigma_{n}^{2}}, \forall m \in \mathcal{M}, k \in \mathcal{K}_{m}, n \in \mathcal{N}
$$

where $\gamma_{k}^{D 2 D_{m}}$ denotes the channel quality of $k$-th cluster member in $m$-th D2D multicast cluster. $P_{n}^{C U}$ represents the transmit power of $\mathrm{CU} n$ who shares uplink RB $n$ together. $G_{m, k}^{D 2 D}$ and $G_{n, k}^{C 2 D}$ stand for the channel gain between the cluster head and cluster member $k$ in the $m$-th $\mathrm{D} 2 \mathrm{D}$ cluster, between interfering CU $n$ to member $\mathrm{UE} k$, respectively. $\sigma_{n}^{2}$ denotes the noise power.

Similarly, the channel quality of a CU $n$ is calculated as:

$$
\gamma_{n}^{C U}=\frac{G_{n, B S}^{C U}}{\sum_{m=1}^{M} x_{m, n} P_{m}^{D 2 D_{m}} G_{m, B S}^{D 2 C}+\sigma_{n}^{2}}, \forall m \in \mathcal{M}, n \in \mathcal{N}
$$

Here, $P_{m}^{D 2 D_{m}}$ stands for the transmit power of the cluster head in $m$-th D2D cluster who reuses the $\mathrm{RB}$ of $\mathrm{CU} n . G_{n, B S}^{C U}$ and $G_{m, B S}^{D 2 C}$ represent the link gain from $C U n$ to the BS and from co-channel cluster head to the BS, respectively.

According to [6], it is commonly assumed that the transmission rate of a multicast is determined by the user with the worst channel condition. Combined with the scenario shown in Figure 1, for the $m$-th D2D cluster, the transmission rate achieved at RB $n$ is given by

$$
\gamma_{m, n}^{D 2 D}=\min _{\forall k \in \mathcal{K}_{m}} \gamma_{k}^{D 2 D_{m}}
$$


Therefore, we can calculate the normalized transmission rate of the $m$-th D2D cluster as

$$
r_{m}^{D 2 D}=\sum_{n=1}^{N} x_{m, n} \log _{2}\left(1+P_{m}^{D 2 D_{m}} \gamma_{m, n}^{D 2 D}\right)
$$

As a result, the transmission rate of $m$-th D2D cluster is expressed as

$$
R_{m}^{D 2 D}=\left|\mathcal{K}_{m}\right| r_{m}^{D 2 D}
$$

Similarly, we can formulate the normalized transmission rate for a $\mathrm{CU} n$ which use $\mathrm{RB} n$ as

$$
R_{n}^{C U}=\log _{2}\left(1+P_{n}^{C U} \gamma_{n}^{C U}\right)
$$

In order to guarantee the quality of service (QoS) requirement of each UE, a threshold is set. Specifically, for the $m$-th D2D multicast cluster, the above requirement is expressed as:

$$
P_{m}^{D 2 D_{m}} \gamma_{m}^{D 2 D} \geq \Gamma_{\min }^{D 2 D}
$$

The above expression is explained as in order to ensure reliable transmission, the signal-to-interference-and-noise ratio (SINR) of the user who has the worst channel condition among a D2D multicast cluster should be above certain threshold. Again, for a $\mathrm{CU}$, this requirement is set as

$$
P_{n}^{C U} \gamma_{n}^{C U} \geq \Gamma_{\min }^{C U}
$$

where $\Gamma_{\min }^{D 2 D}$ and $\Gamma_{\min }^{C U}$ represent the minimum SINR threshold to ensure reliable D2D multicast and cellular transmission specified by the system.

Combined with the maximum transmit power constraints for CUs and D2D clusters and by substituting expressions (2) and (3) into (8) and (9), the transmit power range of a cluster head and a $\mathrm{CU}$ is expressed as

$$
\left\{\begin{array}{c}
\frac{\Gamma_{\min }^{D 2 D}}{\min \frac{G_{m, k}^{D 2 D}}{\sum_{n=1}^{N} x_{m, n} P_{n}^{P U} G_{G, k}^{C 2 D}+\sigma_{n}^{2}}} \leq P_{m}^{D 2 D_{m}} \leq P_{\max }^{D 2 D}, \forall m \in \mathcal{M}, k \in \mathcal{K}_{m} \\
\frac{\Gamma_{\min }^{C U}}{\frac{G_{n, B S}^{C U}}{\sum_{m=1}^{M} x_{m, n} P_{m}^{D 2 D} D_{m} G_{m, B S}^{D 2 C}+\sigma_{n}^{2}}} \leq P_{n}^{C U} \leq P_{\max }^{C U}, \forall m \in \mathcal{M}, n \in \mathcal{N}
\end{array}\right.
$$

where $P_{\max }^{C U}$ and $P_{\max }^{D 2 D}$ represent the maximum allowed transmit power of a $\mathrm{CU}$ and a D2D user, respectively. From expression (10), we can deduce that besides the channel condition factor, the transmit power of the cluster head as well as the CU who shares the RB resource with a D2D multicast cluster are intertwined. In order to guarantee reliable transmission, the transmit power of different kinds of UEs should be considered.

Given the limited energy capacity of each device, the objective of this paper is to maximize the overall energy efficiency of all D2D multicast clusters, which is also a hottest research aspect. According to [13], the energy-efficiency (EE) of a single D2D multicast cluster $m$ can be expressed as

$$
\eta_{m}=\frac{R_{m}^{D 2 D}}{P_{m}^{D 2 D_{m}}}
$$

Combing Equations (1)-(11) and since there are $M$ D2D multicast clusters, the energy efficiency of all the D2D multicast clusters is defined as the ratio of total D2D multicast transmission data rates to the overall consumed power of all clusters. Consequently, the energy efficiency optimization problem can be expressed as 


$$
\max _{\forall m \in \mathcal{M}} \eta_{\text {all }}=\frac{\sum_{m=1}^{M} R_{m}^{D 2 D}}{\sum_{m=1}^{M} P_{m}^{D 2 D_{m}}}
$$

s.t.

$$
\begin{aligned}
& P_{m}^{D 2 D_{m}} \gamma_{m}^{D 2 D} \geq \Gamma_{\min }^{D 2 D}, \forall m \in \mathcal{M}, n \in \mathcal{N} \\
& P_{n}^{C U} \gamma_{n}^{C U} \geq \Gamma_{\min }^{C U} \\
& \sum_{n=1}^{N} x_{m, n} \leq 1, \forall m \in \mathcal{M} \\
& \sum_{m=1}^{M} x_{m, n} \leq 1, \forall n \in \mathcal{N} \\
& \frac{\Gamma_{\min }^{D 2 D}}{\min \frac{G_{m, k}^{D 2 D}}{\sum_{n=1}^{N} x_{m, n} P_{n}^{C U} G_{n, k}^{C 2 D}+\sigma_{n}^{2}}} \leq P_{m}^{D 2 D_{m}} \leq P_{\max }^{D 2 D}, \forall m \in \mathcal{M} \\
& \frac{\Gamma_{\min }^{C U}}{\frac{G_{n, B S}^{C U}}{\sum_{m=1}^{M} x_{m, n} P_{m}^{D 2 D_{m}} G_{m, B S}^{D 2 C}+\sigma_{n}^{2}}} \leq P_{n}^{C U} \leq P_{\max }^{C U}, \forall m \in \mathcal{M}, n \in \mathcal{N}
\end{aligned}
$$

where $\eta_{\text {all }}$ represents the overall energy-efficiency of D2D clusters. Constraints (12a) and (12b) define the minimum SINR requirement. Constraints (12c) and (12d) ensure that each D2D cluster reuses the RBs of CU at most once. Constraints (12e) and (12f) ensure that the transmit power both D2D users and CUs fall into certain range.

According to Equation (12), in order to improve the total energy-efficiency, the possible solution is either to decrease the transmit power or to increase the aggregate transmission rate. This is interpreted as we have to find the optimal resource reuse relationship $x_{m, n}$ between D2D clusters and CUs, as well as to determine the optimal transmit power of both CUs and D2D clusters which also guarantees the minimum SINR requirement.

As a matter of fact, when D2D multicast transmission undelaying with a cellular network, the resource allocation method and power allocation strategy are actually interacted with each other [14]. Once the resource reuse relationship between CUs and D2D clusters varies, the transmit power of each UE will also be influenced owing to changing interference condition. On the other hand, if the transmit power of different UE alters, the interference condition between co-channel CUs and D2D clusters will also change, which conversely affects the resource assignment results. In addition, the existence of integer assignment variable $x_{m, n}$ makes the optimization problem more complicated.

Consequently, the above optimization problem in (12) is a non-convex optimization problem which is proved to be a NP-Hard problem and there are no efficient solutions [15]. Moreover, when the problem size increases, the computational complexity also increases exponentially. A possible solution for above problem might be using the bipartite matching based optimal resource allocation scheme, as suggested by [16]. However, such scheme is actually based on exhaustive searching method, which results in high computational complexity. In order to deal with this challenge, we propose a heuristic resource allocation and power control algorithm to balance the system performance and complexity.

\section{Proposed Heuristic Energy-Efficient Resource and Power Allocation Algorithm}

According to (12), we can deduce that in order to improve energy-efficiency of all the D2D multicast clusters, we have to either decrease the transmit power or to increase the aggregate transmission rate. However, due to the fraction form of the objective function built in (12), it is a non-convex optimization problem. Hence, it is difficult to obtain the optimal solution directly. In the 
following part, we will first solve the overall energy-efficiency optimization problem by adopting a heuristic resource allocation strategy.

Firstly, we consider how to increase the numerator of expression (12). From the resource assignment point of view, in order to improve the aggregate D2D throughput, higher values of SINR are desirable. From constraints (12a)-(12f), we can infer that a smaller value of $G_{n, m}^{C 2 D}$ means less interference from co-channel CU $n$ to D2D cluster $m$, which will result in higher $\gamma_{m}^{D 2 D}$ and D2D throughput. Hence, the fundamental idea of the proposed scheme is to pick up the CU who generates less interference to the co-channel D2D cluster. To achieve that target, we build a channel state information (CSI) matrix $\mathbf{G}_{\mathbf{M} \times \mathbf{N}}^{\mathrm{C} 2 \mathrm{D}}$, which is composed of estimated channel gain information of each D2D cluster from the interfering $C U$ respectively, where $G_{\mathbf{M} \times \mathbf{N}}^{C 2 D}$ is expressed as

$$
\mathbf{G}_{\mathbf{M} \times \mathbf{N}}^{\mathbf{C 2 D}}=\left[\begin{array}{c}
G_{1,1}^{C 2 D}, G_{2,1}^{C 2 D}, \ldots, G_{N, 1}^{C 2 D} \\
G_{1,2}^{C 2 D}, G_{2,2}^{C 2 D}, \ldots, G_{N, 2}^{C 2 D} \\
\vdots \\
G_{1, M}^{C 2 D}, G_{2, M}^{C 2 D}, \ldots, G_{N, M}^{C 2 D}
\end{array}\right]
$$

In the matrix $\mathrm{G}_{\mathbf{M} \times \mathbf{N}}^{\mathrm{C} 2 \mathrm{D}}$, each element stands for the CSI value from the co-channel $\mathrm{CU}$ to the corresponding D2D multicast cluster. We assume that the CSI between each CU and each D2D cluster can be obtained by each cluster head individually. Actually, such information can be initially obtained through information change between each cluster head and D2D receivers. Then, the CSI information between each cluster and each CU can be gathered at the BS side through control information exchange. Consequently, we can find the minimum $G_{n, m}^{C 2 D}, n \in \mathcal{N}, m \in \mathcal{M}$ in each row so as to pair up the $C \mathrm{U}$ which brings the least interference to the D2D multicast cluster. By doing the same procedure for each D2D multicast cluster, the optimal resource assignment between CU and the corresponding D2D multicast cluster can be decided.

After picking out the cellular resource for each D2D cluster, the next step is to decrease the denominator of expression (12). This is interpreted as to determine the minimum transmit power of each CU and D2D cluster respectively, which also satisfies the minimum SINR threshold requirement. Suppose a CU $n$ shares RB resource with a D2D cluster $m$ after resource pairing process. By using the Equations (8) and (9) and substituting $\gamma_{n}^{C U}$ and $\gamma_{m}^{D 2 D}$ from expression (3) and (4) respectively, we can determine the optimal transmit power of a D2D cluster and a CU which can guarantee reliable transmission as follows

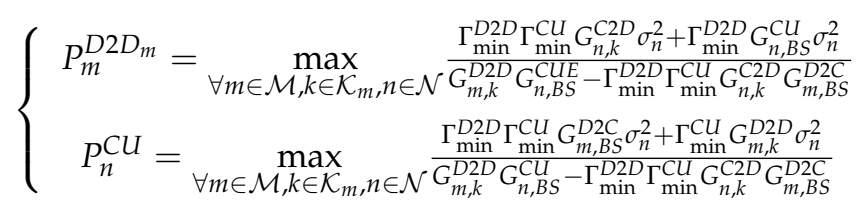

Combined with the expressions given in (14), if the calculated transmit power of both CUs and D2D users obtained in (14) do not exceed the maximum allowed transmit power, CU $n$ is finally chosen as the resource sharing partner for D2D cluster $m$. By substituting (14) into the optimization function (12), we can determine the minimum transmit power of different D2D multicast cluster so as to maximize the overall energy-efficiency of D2D clusters. On the contrary, if the calculated transmit power of both CUs and D2D users exceed the maximum allowed values, then CU $m$ will be removed from the available resource assignment list and we will try the next available $\mathrm{CU}$ according to the matrix $\mathrm{G}_{\mathbf{M} \times \mathbf{N}}^{\mathrm{C} 2 \mathrm{D}}$. The pseudo code of the proposed energy-aware resource allocation and power control algorithm is given in Algorithm 1. 


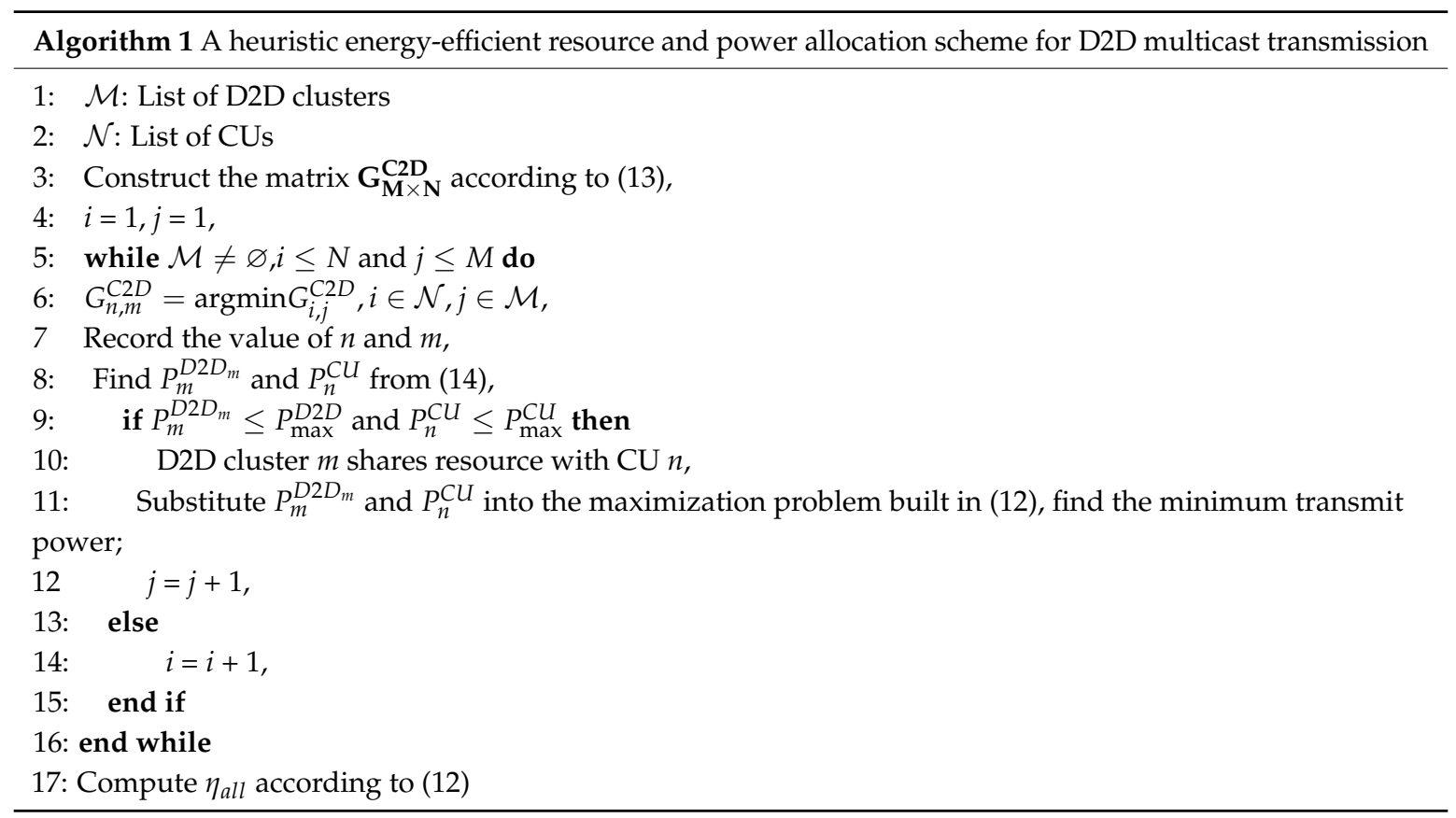

In the proposed scheme, in order to maximize the overall energy-efficiency of D2D multicast clusters, we first search for the optimal RB assignment between CUs and D2D clusters. This leads to increased total data rates of D2D multicast transmission. Then, combined with the minimum SINR requirement, we find out the minimum transmit power of both CUs and D2D clusters to ensure reliable D2D multicast transmission. Hence, the maximization problem built in expression (12) is solved by a two-step way, where the computational complexity of the proposed strategy is $(M N)+\left(M \times f\left|\mathcal{K}_{m}\right|\right)$. This is because in the worst case, the maximization problem will be solved in $M$ times. Here, $f\left|\mathcal{K}_{m}\right|$ represents the size of each D2D multicast cluster. It can be seen that, compared with exhaustive searching based methods, (e.g., such as [16], where the complexity is $\left.\left(M^{3}\right)+\left(M \times N \times f\left|\mathcal{K}_{m}\right|\right)\right)$, the proposed heuristic solution can considerably reduce the computational complexity.

\section{Simulation Results}

In this section, numerical results are provided to demonstrate the performance of our proposed strategy. We use the clustered distribution model adopted in [17], where a $400 \mathrm{~m} \times 400 \mathrm{~m}$ square area is used to simulate the network. Cluster heads are randomly distributed in the simulation area according to the uniform distribution and the D2D users are randomly distributed in the corresponding multicast cluster. The distance-based path loss and shadowing fading are considered for the transmission channel. We still consider the scenario that D2D clusters and CUs share uplink cellular RBs together. Suppose that all available resource is divided into RBs and each CU is allocated with one RB at each scheduling slot. Other related simulation parameters are listed shown in Table 1.

In order to demonstrate the performance of the proposed energy-aware resource and power allocation scheme, three other different algorithms are considered. The first one is the QoS-aware resource allocation scheme proposed in [7], which aims at minimizing the interference among D2D multicast cluster and CU through resource allocation. Moreover, the power control policy in [7] assumes that both CU and D2D cluster transmit at the maximum power when the channel condition is good enough. The second one is the cluster based scheme proposed in [18], which employs social information to facilitate file transfer process. In the absence of resource pairing scheme between D2D cluster and CU, the resource assignment between D2D cluster and CU in [18] is assumed to be chosen randomly while power control method is not applied. The third one is the energy-efficient scheme proposed in [14], which aims at improving the energy efficiency multicast transmission through proper power control. The power control principle of [14] is similar to our proposed scheme which tries to 
allocate more transmit power to D2D pairs when the channel condition becomes good. For clarity, our proposed scheme is referred to as the "Proposed energy-aware" scheme.

Table 1. The simulation parameters. UE: user equipment; RB: resource blocks; SINR: signal-tointerference-and-noise ratio; CU: cellular users; D2D: Device-to-Device.

\begin{tabular}{ll}
\hline Parameter & Value \\
\hline Cell Radius & $400 \mathrm{~m}$ \\
Total UE number & 500,1000 \\
Spectrum bandwidth & $10 \mathrm{MHz}$ \\
Bandwidth of each RB & $180 \mathrm{KHz}$ \\
The path loss Component $(\alpha)$ & 4 \\
Shadowing & Log-normal fading with standard deviation of $8 \mathrm{~dB}$ \\
Noise power spectrum density $\left(\sigma^{2}\right)$ & $-174 \mathrm{dBm} / \mathrm{Hz}$ \\
Minimum SINR Threshold $\left(\Gamma_{\min }^{D 2 D}, \Gamma_{\min }^{C U}\right)$ & $10 \mathrm{~dB}$ \\
Maximum transmit power of UE $\left(P_{\max }^{C U}, P_{\max }^{D 2 D}\right)$ & $\mathrm{CU}: 23 \mathrm{dBm}, \mathrm{D} 2 \mathrm{D}$ user: $20 \mathrm{dBm}$ \\
D2D cluster radius $(r)$ & $30 \sim 90 \mathrm{~m}$ \\
UE Transmission range & $90 \mathrm{~m}$ \\
\hline
\end{tabular}

Figure 2 illustrates the sum energy efficiency of the D2D multicast cluster with the variation of the D2D cluster radius. From Figure 2 we can see that with the increase of the D2D cluster radius, the energy efficiency performance of our proposed scheme is better than other referenced schemes. This is because the channel gain of the D2D link will decrease with the increase of D2D cluster radius. Hence, a larger transmit power is required for the D2D clusters so as to satisfy the SINR threshold constraint. Accompanied with the increase of D2D cluster radius, our proposed scheme gradually increases the transmit power. This results in the decreased energy efficiency of the D2D cluster. Compared with the scheme in [14], our proposed resource allocation method minimizes the interference from CUs to co-channel D2D receivers, which improves the channel gain of D2D links. Furthermore, it is shown that when there are more UEs in the considered scenarios ( $N$ varies), the overall energy efficiency also increases due to the increased total transmission rate of all D2D multicast clusters.

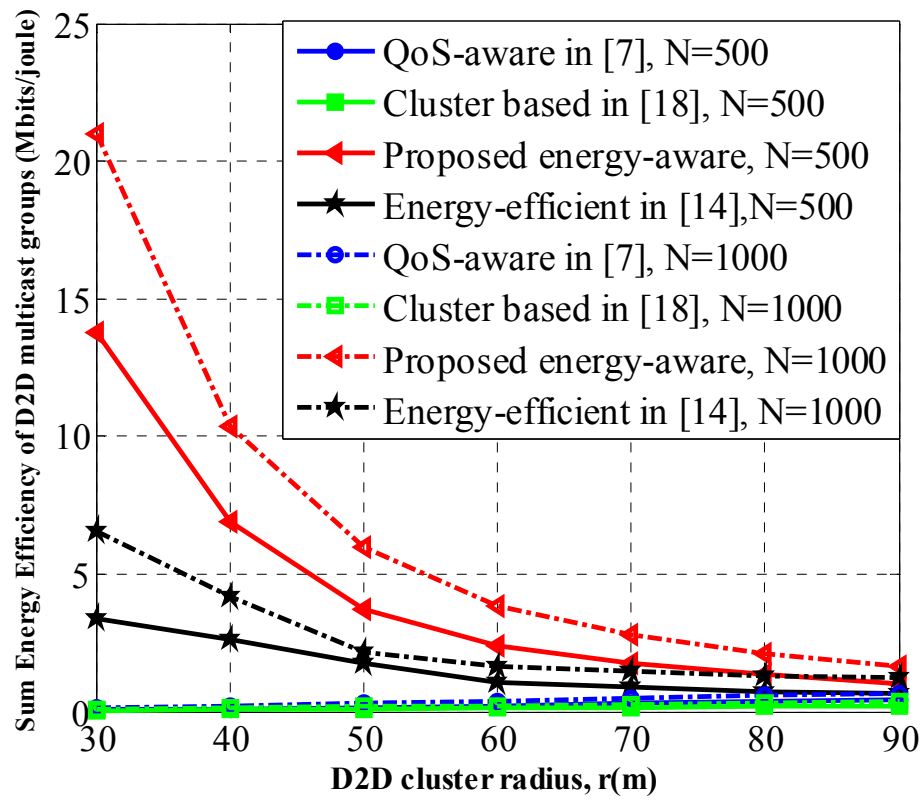

Figure 2. Cluster Energy Efficiency versus D2D cluster radius. QoS: quality of service.

Figure 3 compares the total throughput of D2D multicast clusters of the proposed scheme with three other schemes. From the figure, we can see that the total throughput performance of the proposed 
scheme is initially inferior to that of [7] and gradually outperforms other schemes with the increase of D2D cluster radius. The reason is twofold. Firstly, our proposed scheme aims at maximizing the EE performance of the D2D clusters, which adopts a smaller transmit power when the radius of the D2D multicast group is small. This explains why the total throughput of our proposed scheme is smaller than that of the scheme in [7], which always adopts the maximum transmit power. However, with the increase of D2D cluster radius, our proposed scheme will gradually increase the transmit power of D2D users while decrease the transmit power of co-channel CUs in order to ensure reliable D2D multicast transmission, which leads to increased total throughput. Secondly, compared with schemes in $[14,18]$, the resource relationship of our proposed scheme is based on minimizing the interference of D2D links. As a result, the CU who brings the least interference will be paired up with the D2D multicast cluster accordingly. This explains why our proposed scheme has better performance. Similarly, it is shown that the sum throughput of all four algorithms increases with the increase of user numbers.

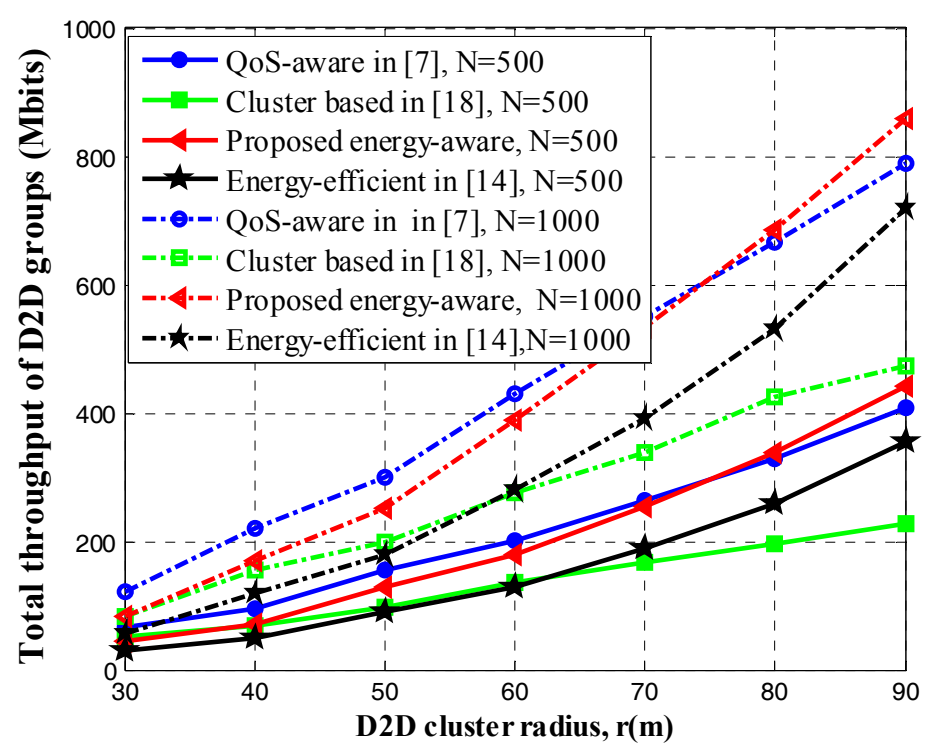

Figure 3. Total Throughput of D2D multicast clusters versus D2D cluster radius.

The impact of energy efficiency on total throughput of D2D multicast groups is shown in Figure 4. It can be observed that the energy efficiency performance of our proposed scheme and the scheme in [14] both decrease with the increase of total throughput. This is explained as when the total throughput increases, according to our proposed power control scheme, the transmit power also increases, which contributes to decreased energy efficiency. Moreover, based on minimizing the interference of D2D clusters, our proposed heuristic algorithm allocates better cellular resource to D2D clusters. Meanwhile, it also decreases the transmit power of co-channel CUs, which contributes to improved EE performance of D2D multicast clusters.

Figure 5 plots the average SINR of D2D multicast clusters with different D2D cluster radius. We assume that there are totally 500 UEs randomly distributed in the simulation area. From the figure, we can infer that for the schemes which include power control scheme, such as our proposed algorithm and the scheme in [14], the SINR distribution does not obviously decrease with the increase of D2D group radius. This is because according to the changing channel conditions and resource reuse relationships, the power control method jointly adjusts the transmission power of both D2D clusters and CUs, which ensures reliable D2D multicast transmission [19]. On the contrary, for the algorithms which do not incorporate power control schemes, such as $[7,18]$, the QoS requirement of D2D multicast clusters in terms of minimum SINR constraint cannot be guaranteed when the channel quality becomes worse. 


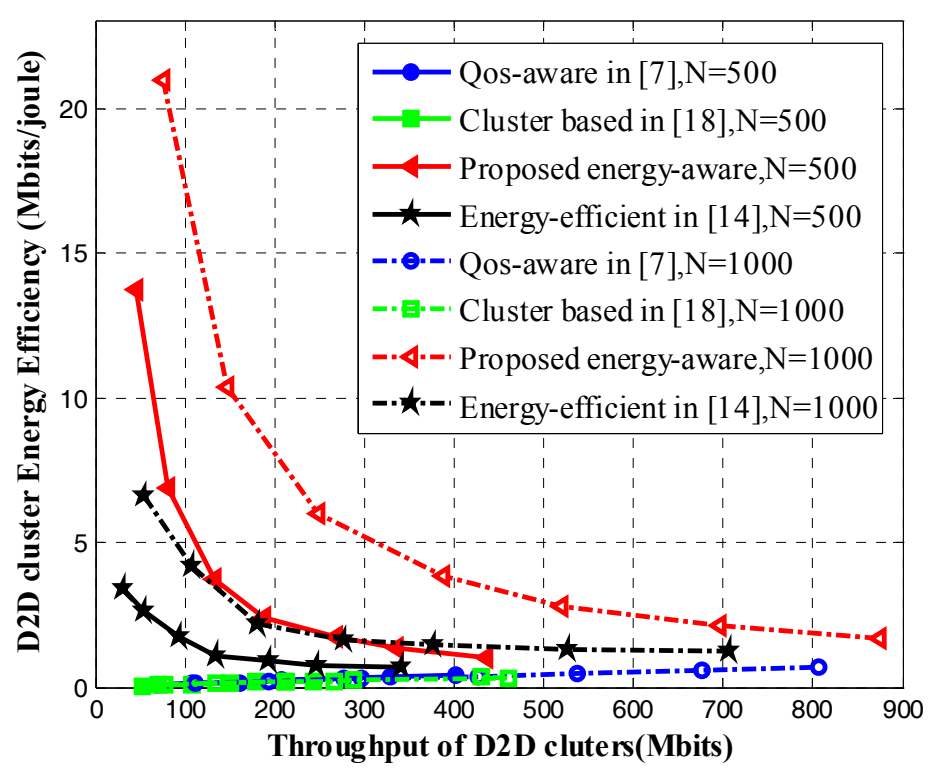

Figure 4. D2D Group Energy Efficiency versus throughput.

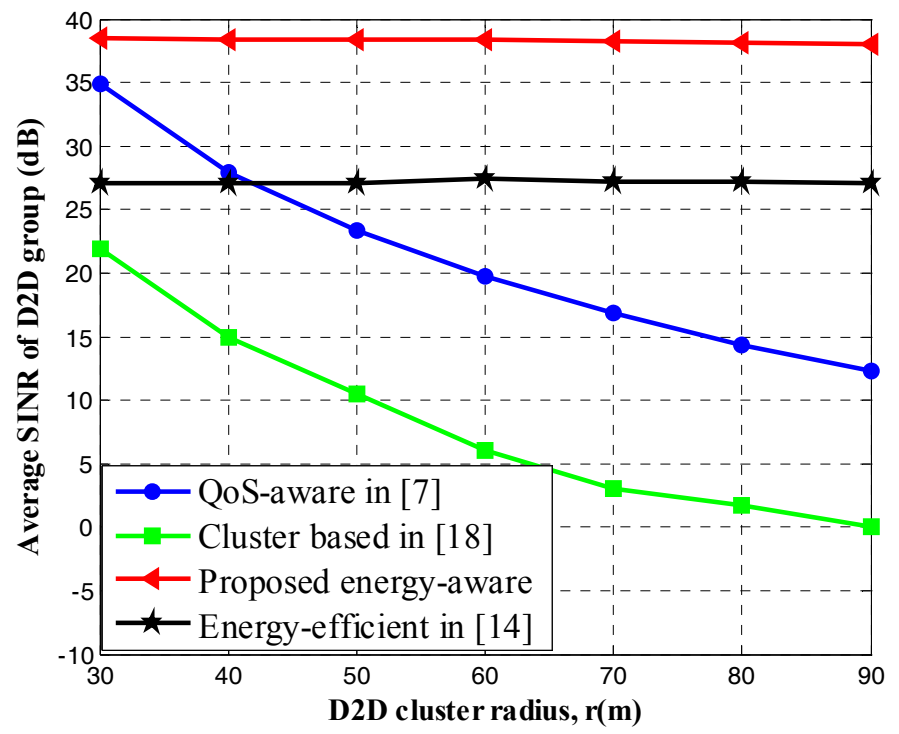

Figure 5. Average D2D group Energy efficiency versus number of group users.

Figure 6 plots the Cumulative Distribution Function (CDF) curve of cellular users' SINR concerning with different schemes. It can be obviously seen that with the introduction of power control scheme, the SINR curves of both [14] and our proposed scheme decrease, while the SINR curves of $[7,18]$ are not severely impacted. This is because our proposed strategy decreases the transmit power of CUs in order to maximize the total energy-efficiency of D2D multicast clusters, which results in deterioration of CU's SINR. However, compared with [14], our resource allocation strategy will pick up a CU who brings the least interference for the D2D multicast cluster. Hence, the co-channel interference caused by underlay D2D transmission will be effectively controlled, which contributes to improved SINR of C-links. From Figure 6, we can also infer that although the link quality of CUs has been affected a little for the proposed scheme. But the performance degradation is still acceptable to CUs because the power allocation strategy considers the minimum SINR constraint of different users. 


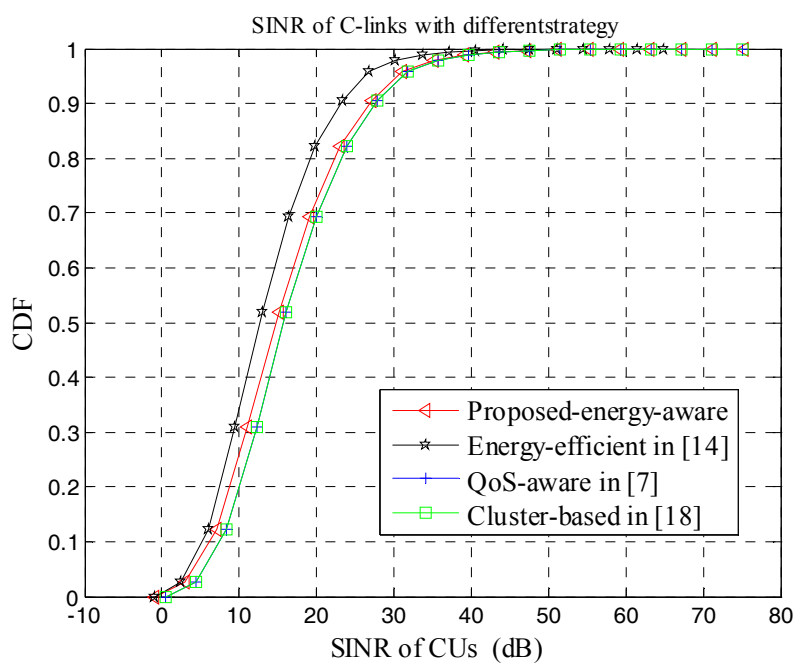

Figure 6. Signal-to-interference-and-noise ratio (SINR) of C-links with different schemes.

\section{Conclusions}

In this paper, we have investigated an energy-efficient resource and power allocation algorithm for multicast D2D communication underlying a cellular network. The goal of this paper is to maximize the overall energy efficiency of D2D multicast groups through appropriate resource allocation and a power control scheme, while maintaining the SINR requirements of both CUs and D2D clusters. A heuristic resource and power control algorithm is then proposed to solve the above problem with less complexity. It is shown by simulation that the energy efficiency of D2D multicast clusters with the proposed scheme can be improved significantly compared with conventional resource allocation schemes. In future, we plan to extend this work to the multiple multicast problem which means that several multicast clusters are allowed to reuse the same RB of a CU to further improve the spectrum efficiency.

Acknowledgments: This work is supported by the National Natural Science Foundation of China (No. 61501371), National Science and Technology Major Project of the Ministry of Science and Technology of China(project number: 2017ZX03001012-005). The international Exchange and Cooperation Projects of Shaanxi Province (project number: 2016KW-046).

Author Contributions: Fan Jiang conceived and wrote the paper, Honglin Wang and Hao Ren designed and conducted comprehensive computer simulations, Shuai $\mathrm{Xu}$ helped Fan Jiang to conduct the theory analysis.

Conflicts of Interest: The authors declare no conflict of interest.

\section{References}

1. IMT-2020 (5G) Promotion Group. White Paper on 5G Wireless Technology Architecture. Available online: http:/ / www.imt-2020.cn/zh (accessed on 15 May 2015).

2. Tehrani, M.N.; Uysal, M.; Yanikomeroglu, H. Device-to-device communication in 5G cellular networks: Challenges, solutions and future directions. IEEE Commun. Mag. 2014, 52, 86-92. [CrossRef]

3. Wei, L.L.; Hu, R.Q.; Qian, Y. Enable device-to-device communications underlying cellular networks: Challenges and research aspects. IEEE Commun. Mag. 2014, 52, 90-96. [CrossRef]

4. Condoluci, M.; Militano, L.; Araniti, G. Multicasting in LTE-A networks enhanced by device-to-device communications. In Proceedings of the 2013 IEEE Globecom Workshops (GC Workshops), Atlanta, GA, USA, 9-13 December 2013; pp. 567-572.

5. Shang, B.; Zhao, L.; Chen, K.C. Operator's Economy of Device-to-Device Offloading in Underlaying Cellular Networks. IEEE Commun. Lett. 2017, 21, 865-868. [CrossRef] 
6. Meshgi, H.; Zhao, D.; Zheng, R. Joint channel and power allocation in underlay multicast device-to-device communications. In Proceedings of the 2015 IEEE International Conference on Communications (ICC), London, UK, 8-12 June 2015; pp. 2937-2942.

7. Bhardwaj, A.; Agnihotri, S. A resource allocation scheme for device-to-device multicast in cellular networks. In Proceedings of the 2015 IEEE 26th Annual International Symposium on Personal, Indoor and Mobile Radio Communications (PIMRC), Hong Kong, China, 30 August-2 September 2015; pp. 1498-1502.

8. Kitagawa, K.; Homma, H.; Suegara, Y. A user selection algorithm for D2D multicast communication underlying cellular systems. In Proceedings of the 2017 IEEE Wireless Communications and Networking Conference (WCNC 2017), San Francisco, CA, USA, 19-22 March 2017; pp. 1-6.

9. Yu, S.; Langar, R.; Wang, X. A D2D-Multicast Based Computation Offloading Framework for Interactive Applications. In Proceedings of the 2016 IEEE Global Telecommunications Conference (GLOBECOM 2016), Washington, DC, USA, 4-8 December 2016; pp. 1-6.

10. Zhao, P.; Feng, L.; Yu, P. Resource allocation for energy-efficient Device-to-Device multicast communication. In Proceedings of the 2016 19th International Symposium on Wireless Personal Multimedia Communications (WPMC 2016), Shenzhen, China, 14-16 November 2016; pp. 518-523.

11. Chen, X.; Pu, L.; Gao, L. Exploiting massive D2D collaboration for energy-efficient mobile edge computing. IEEE Wirel. Commun. 2016, 24, 64-71. [CrossRef]

12. Jiang, L.; Tian, H.; Xing, Z. Social-aware energy harvesting device-to-device communications in $5 \mathrm{G}$ networks. IEEE Wirel. Commun. 2016, 23, 20-27. [CrossRef]

13. Xu, L.; Jiang, C.; Shen, Y. Energy efficient D2D communications: A perspective of mechanism design. IEEE Trans. Wirel. Commun. 2016, 15, 7272-7285. [CrossRef]

14. Guan, N.; Zhou, Y.; Liu, H. An energy efficient cooperative multicast transmission scheme with power control. In Proceedings of the 2011 IEEE Global Telecommunications Conference (GLOBECOM 2011), Houston, TX, USA, 5-9 December 2011; pp. 1-5.

15. Feng, D.; Yu, G.; Xiong, C. Mode switching for energy-efficient device-to-device communications in cellular networks. IEEE Trans. Wirel. Commun. 2015, 14, 6993-7003. [CrossRef]

16. Feng, D.Q.; Lu, L.; Wu, Y.Y.; Li, G.Y.; Li, S.Q. Device-to-device communications underlying cellular Networks. IEEE Trans. Commun. 2013, 61, 3541-3551. [CrossRef]

17. Peng, B.; Peng, T.; Liu, Z. Cluster-based multicast transmission for Device-to-Device (D2D) communication. In Proceedings of the 2013 IEEE 78th Vehicular Technology Conference (VTC Fall), Las Vegas, NV, USA, 2-5 September 2013; pp. 1-5.

18. Zhang, G.; Yang, K.; Chen, H.H. Socially aware cluster formation and radio resource allocation in D2D networks. IEEE Wirel. Commun. 2016, 23, 68-73. [CrossRef]

19. Ren, Y.; Liu, F.; Liu, Z.; Ji, Y. Power control in D2D-based vehicular communication networks. IEEE Trans. Veh. Technol. 2015, 64, 5547-5562. [CrossRef]

(C) 2017 by the authors. Licensee MDPI, Basel, Switzerland. This article is an open access article distributed under the terms and conditions of the Creative Commons Attribution (CC BY) license (http:/ / creativecommons.org/licenses/by/4.0/). 